\title{
Corela
}

Cognition, représentation, langage

19-1 | 2021

Vol. $19, \mathrm{n}^{\circ} 1$

\section{Étude de l'activité de définition de noms chez des enfants de 9-11 ans issus de classes sociales contrastées}

Thierry Chanselme

\author{
(2) OpenEdition \\ Journals \\ Electronic version \\ URL: https://journals.openedition.org/corela/12885 \\ DOI: 10.4000/corela.12885 \\ ISSN: 1638-573X \\ Publisher \\ Cercle linguistique du Centre et de l'Ouest - CerLICO
}

\section{Electronic reference}

Thierry Chanselme, "Étude de l'activité de définition de noms chez des enfants de 9-11 ans issus de classes sociales contrastées", Corela [Online], 19-1 | 2021, Online since 07 July 2021, connection on 13 July 2021. URL: http://journals.openedition.org/corela/12885 ; DOI: https://doi.org/10.4000/corela. 12885

This text was automatically generated on 13 July 2021.

\section{(c) (i) (2) (2)}

Corela - cognition, représentation, langage est mis à disposition selon les termes de la licence Creative Commons Attribution - Pas d'Utilisation Commerciale - Partage dans les Mêmes Conditions 4.0 International. 


\title{
Étude de l'activité de définition de noms chez des enfants de 9-11 ans issus de classes sociales contrastées
}

\author{
Thierry Chanselme
}

\section{Introduction}

1 Cette recherche vise à rendre compte de l'organisation du contenu sémantique de définitions de noms produites par des enfants âgés de 9 à 11 ans, et des stratégies de construction de ces définitions en fonction du milieu socio-économique d'origine. Pour mener cette analyse, nous allons d'abord faire un bref état des lieux sur l'activité définitionnelle en convoquant les recherches, principalement psycholinguistiques, qui ont exploré les productions orales des enfants dans le cadre de tâches de définition. Les apports scientifiques, encore peu nombreux, qui ont investigué les rapports entre le milieu social et la production de définitions seront également présentés. Par la suite, nous décrirons notre protocole et nos choix méthodologiques puis confronterons nos résultats à la lumière des études existantes en tâchant de montrer ce que notre étude apporte à la réflexion sur la notion et sur l'activité de définition chez l'enfant.

\section{Tâche de définition et vocabulaire profond}

Il existe deux types de démarches pour approcher scientifiquement le concept de vocabulaire et son acquisition chez l'enfant. La première méthode utilise des tâches d'identification ("Qu'est-ce que c'est? ») ou de reconnaissance (« Montre-moi ... ») à partir d'un matériel visuel (imagiers). La seconde approche mobilise des tâches de définition de mots ("Qu'est-ce qu'un(e)...? ?) induisant une réponse orale ou écrite. Les recherches sur l'acquisition du vocabulaire montrent que ce concept est multidimensionnel (Pan, 2012, Benelli \& al., 1988). Cette acquisition ne se réduit pas au nombre de mots connus (étendue lexicale) mais s'intéresse aussi au degré de compréhension (vocabulaire 
profond). Les tâches classiques d'identification ou de reconnaissance à partir d'imagiers (Hadley \& al., 2015) ne permettent pas d'estimer la compréhension lexicale. La distinction entre étendue (mots connus) et profondeur (mots compris) est dérivée des modèles du lexique mental (Ouellette, 2006). Les enfants stockent d'abord une représentation phonologique des mots dans leur mémoire lexicale sans totalement en comprendre la signification (Lahey, 1988). Au fil du temps, la signification va s'affiner, pour constituer la connaissance profonde du vocabulaire. La tâche de définition reste le moyen le plus opérationnel pour approcher la connaissance de la signification des mots, c'est-à-dire le vocabulaire profond (Nippold, 1995).

\subsection{La tâche de définition : une activité métalinguistique socialement située}

3 Dans notre perspective, la notion de codage définitionnel (Salazar Orvig \& al., 1988) chez l'enfant, concerne l'ensemble des mécanismes socio-cognitifs par lesquels celui-ci va construire une signification des mots. En pratique, les tâches définitionnelles demandent aux enfants d'expliquer plus que de définir (Cravatte, 1980) : ainsi visentelles à vérifier la maîtrise du sens plutôt que la connaissance des caractéristiques formelles de la définition « dictionnariste ». Pour autant, les stratégies pour répondre à la question "Qu'est-ce qu'un(e) ... » procèdent de règles d'organisation spécifiques bien que rarement enseignées. Ces mécanismes définitionnels sont à la fois cognitifs et sociaux : ils sont d'abord et avant tout sous l'influence des connaissances disponibles et mobilisables sous forme verbale mais également à des degrés divers, ces mécanismes sont soumis à un ensemble de conventions, correspondant aux normes sociales implicites du genre définitionnel, dont l'école est le principal, pour ne pas dire le seul lieu d'expérience (Snow, 1990).

4 L'activité définitionnelle implique une prise de distance et une activité réflexive sur sa langue. C'est cette composante métalinguistique qui pourrait en partie expliquer les difficultés que rencontrent les plus jeunes enfants. En dépit de compétences catégorielles, de logique d'inclusion de classe, d'organisation taxonomique de son système catégoriel (Simoes Loueiro, 2015), le jeune enfant persiste longtemps à fournir des informations autres qu'autoréférencées et centrées sur l'accessoire, le particulier, la caractéristique isolée (Salazar Orvig \& al., 1988, Snow \& al., 1991, Benelli \& al., 2006).

5 Très tôt, vers 4 ans, l'enfant sait que le chat est un animal mais c'est beaucoup plus tard dans son développement qu'il sera capable de mobiliser la catégorie générique «animal» dans une activité définitionnelle (McGhee-Bidlack, 1991, Watson, 1985, Anglin, 1970).

\subsection{Développement du vocabulaire et classes sociales}

6 À l'école, la référence à l'origine sociale des élèves est récurrente pour tenter d'expliquer les difficultés langagières et scolaires.

7 Une étude devenue classique de Hart \& al. (1995) a montré des différences quant à l'étendue du vocabulaire selon le milieu socio-économique des parents, et ceci dès le début de l'acquisition de la parole. Dans leur enquête, un effet de la fréquence et de la qualité des interactions langagières familiales précoces est mis en évidence. 
8 À partir des données de l'enquête ELFE sur un suivi longitudinal de 18000 enfants français, Grobon \& al. (2019) ont récemment montré, par la méthode du compte-rendu parental, que dès deux ans, le nombre de mots produits est marqué par un fort gradient socio-économique selon le revenu du ménage ou le diplôme des parents. Des analyses croisées tendent à soutenir que ces différences s'atténuent lorsque les enfants fréquentent un mode de garde en dehors de la famille (crèche, assistante maternelle).

Les approches qui établissent de fortes corrélations entre le milieu socio-économique des enfants et le développement des compétences lexicales ont parfois été accusées de fatalisme socio-culturel (Snow, 2017). Si elles ont le mérite de pointer une inégalité de destin majeure (Hoff, 2003, Fernald \& al., 2015), elles ne permettent pas de comprendre les mécanismes par lesquels cette inégalité se construit ou encore quels aspects particuliers de la compétence lexicale sont impliqués et lesquels ne le sont pas. Comment expliquer, par exemple, les réussites de certains enfants issus de milieux sociaux pourtant très défavorisés économiquement ou carencés sur le plan linguistique?

10 Le protocole de la présente étude vise à examiner certains aspects du processus définitionnel et de construction de la signification des mots. Cette recherche vise en particulier à caractériser la nature catégorielle, fonctionnelle ou perceptive des informations proposées dans les définitions et la façon dont les enfants combinent ces différents types d'information.

\subsection{Hypothèses}

Nous testerons trois hypothèses.

12 Dans un premier temps, nous souhaitons examiner, à partir de nos données, l'hypothèse mainte fois testée (Hart \& al., 1995, Snow, 2017) selon laquelle le nombre de mots qu'un enfant est capable d'expliquer est, plus encore que d'autres domaines cognitifs, corrélé avec son milieu socio-économique. Plus la catégorie sociale est élevée plus le nombre de mots de vocabulaire correctement définis est élevé (voir aussi Mercy \&al., 1982).

13 Nous proposerons dans un deuxième temps l'hypothèse selon laquelle les sujets de notre échantillon mobiliseraient des schémas de réponses de nature différente selon leur origine sociale. Si l'on se réfère aux résultats obtenus antérieurement (e.g. Ehrlich \& al., 1978), nous attendons que les élèves de milieux socio-économiques favorisés développent plus souvent des patterns privilégiant un codage par la catégorie puis par la fonction et/ou les traits descriptifs accessibles à la perception. En revanche, nous attendons des sujets de milieux défavorisés qu'ils produisent plus fréquemment un codage définitionnel axé sur la fonction et les traits accessibles à la perception et moins souvent un codage par la catégorie supra-ordonnée.

14 Enfin, notre troisième hypothèse vise à repérer un lien éventuel entre la première information fournie par les enfants et leur milieu socio-économique d'origine. Dans la continuité des recherches précédentes (Al-Issa, 1969, Ehrlich \& al., 1978) nous examinerons l'hypothèse selon laquelle les enfants issus de milieux défavorisés auraient tendance à mobiliser en premier lieu un codage perceptif ou fonctionnel tandis que les sujets issus de milieux socio-économiques favorisés auraient davantage tendance à activer prioritairement une information catégorielle. 
La mise en tension de ces trois hypothèses a pour but de questionner le rôle de la classe sociale sur l'acquisition du vocabulaire profond dans ses dimensions quantitatives (mesurées par la performance au test de vocabulaire) et qualitatives (caractérisation des comportements ou stratégies de codage définitionnel). En d'autres termes, comment et avec quelle ampleur les différences de performances selon la classe sociale lors d'une tâche de définition se traduisent-elles dans les comportements de codage définitionnel?

\section{Protocole pour l'étude de l'activité définitionnelle auprès d'enfants de 9-11 ans}

16 Nous retrouvons ici l'ensemble des variables étudiées, les hypothèses associées et le déroulement de la passation.

\subsection{Présentation des mesures} des définitions.

\subsection{La tâche de définition}

La tâche de vocabulaire de la WISC-IV a été choisie pour ses bonnes qualités psychométriques. La WISC-IV (Echelle d'Intelligence de Wechsler pour Enfants) est un instrument clinique d'application individuelle qui sert à évaluer les performances cognitives des enfants de 6 ans et 0 mois à 16 ans et 11 mois. Les notes brutes obtenues à chacune des épreuves sont rapportées à l'âge des enfants par tranches de trois mois et transformées en notes standard étalonnées, pour tenir compte de l'âge réel. L'épreuve de vocabulaire est conçue pour évaluer la connaissance approfondie ou non d'un certain nombre de mots. Elle est constituée de 36 items dont quatre items en images et 32 items verbaux (mots). La consigne [«Qu'est-ce qu'un(e)...], est présentée oralement 
et le mot inducteur est également présenté par écrit dans un livret. Les réponses orales des sujets sont reportées avec soin, par écrit, par le psychologue, sur le cahier de passation. Pour cette recherche, nous focaliserons nos analyses sur les neuf premiers noms de l'épreuve, qui sont les suivants: parapluie, horloge, vache, gant, alphabet, nénuphar, bicyclette, île, cambrioleur. Seul l'item courrier électronique a été retiré de la liste en raison du fort taux de non-réponses. Les verbes et les adjectifs seront exclus de nos analyses. L'administration de l'épreuve est individuelle. Elle s'est déroulée dans les écoles. La durée de passation de l'épreuve de vocabulaire est d'environ $15 \mathrm{mn}$.

\subsection{Origine sociale des élèves}

Les recherches en sciences sociales et en psychologie utilisent principalement trois indicateurs pour rendre compte de la classe sociale : les revenus, le niveau de diplôme et la profession des parents (e.g., Kraus \& al., 2012). Nous avons opté pour ce dernier, l'emploi occupé, qui renseigne à la fois sur les ressources économiques et culturelles des individus. A la suite d'autres études (e.g., Goudeau \& al., 2017, Croizet \& al., 1998) nous avons construit notre variable classe sociale sur la base de la nomenclature des professions et catégories socio-professionnelles (PCS) de l'Institut national de la statistique et des études économiques (INSEE). Nous avons effectué une catégorisation de notre variable en trois modalités: classe favorisée, classe moyenne, classe défavorisée.

\subsection{Population}

Notre échantillon est constitué de 63 élèves de 9 à 11 ans scolarisés dans 23 écoles publiques rurales et urbaines françaises, recouvrant un large spectre sociodémographique. Parmi ces élèves, 21 sont issus de milieux populaires défavorisés, 21 sont issus de la classe moyenne, et 21 appartiennent à un milieu plutôt aisé sur le plan socio-économique. Les élèves de notre échantillon ont été orientés par leur enseignant(e) vers le psychologue pour un bilan psychométrique, principalement en raison de difficultés scolaires ponctuelles. Tous ont le français pour langue maternelle, aucun ne souffre de problèmes auditifs ou de troubles spécifiques du langage oral ou du développement intellectuel. L'effectif répondant à ces critères étant de 21 pour la classe défavorisée, nous avons, afin d'équilibrer les groupes, tiré au hasard 21 sujets dans les catégories sociales moyennes et favorisées, parmi ceux qui répondaient également à ces critères.

\subsection{Méthode de traitement des productions}

Chaque information contenue dans une définition a été codée selon sa nature perceptive (informations descriptives, d'apparence, traits physiques...), fonctionnelle (informations sur l'usage, l'utilité de l'objet) ou catégorielle (informations sur l'appartenance à une catégorie générique). Depuis les travaux de Al-Issa (1969), puis de Ehrlich \& al. (1978), l'analyse des définitions de noms permet de repérer trois types d'énoncés : catégoriels, fonctionnels et perceptifs. Ces trois types semblent pouvoir contenir l'ensemble des possibilités de codage définitionnel. Nos propres analyses le 
confirment : toutes les unités sémantiques examinées ont pu être rapportées à l'un ou l'autre de ces trois types d'énoncés définitionnels.

\subsection{Rôle de la classe sociale pour six épreuves représentatives du WISC-IV}

Les notes standard moyennes obtenues par 63 sujets issus de trois catégories sociales différentes ont été comparées pour 6 épreuves de l'échelle de Wechsler. Ces épreuves évaluent différents facteurs du fonctionnement cognitif, tels que le fonctionnement verbal (Vocabulaire), le traitement de l'information visuo-spatiale (Cubes), le raisonnement inductif non-verbal (Matrices), la mémoire de travail auditive (Mémoire des chiffres), la vitesse de traitement (Code, Symboles).

Tableau 1 : Note standard moyenne à 6 épreuves du WISC-4 par catégorie sociale

\begin{tabular}{|l|l|l|l|}
\hline & Classe défavorisée & Classe moyenne & Classe favorisée \\
\hline $\begin{array}{l}\text { Vocabulaire } \\
\text { ANOVA-(2)=17,2, }<.001\end{array}$ & 6,14 & 8,14 & 12,10 \\
\hline $\begin{array}{l}\text { Cubes } \\
\text { ANOVA-(2) =6,21, p=0.004 }\end{array}$ & 7,14 & 7,38 & 10,24 \\
\hline $\begin{array}{l}\text { Matrices } \\
\text { ANOVA-(2) }=5,52, \mathrm{p}=0.006\end{array}$ & 8,14 & 8,81 & 10,62 \\
\hline $\begin{array}{l}\text { Mém. des chiffres } \\
\text { ANOVA-(2) }=2,42, \mathrm{p}=0.098\end{array}$ & 9,14 & 7,86 & 9,29 \\
\hline
\end{tabular}




\begin{tabular}{|l|l|l|l|}
\hline $\begin{array}{l}\text { Code } \\
\text { ANOVA-(2) }=1,09, \mathrm{p}=0.343\end{array}$ & 10,14 & 9,14 & 8,81 \\
\hline $\begin{array}{l}\text { Symboles } \\
\text { ANOVA-(2) }=0,960, \mathrm{p}=0.389\end{array}$ & 11,14 & 9,24 & 10,38 \\
\hline
\end{tabular}

\begin{tabular}{|l|l|l|l|l|l|}
\hline Anova Vocabulaire & Sum of Squares & df & Mean Square & F & $p$ \\
\hline PCS & 385 & 2 & 192 & 17.2 & $<.001$ \\
\hline Residuals & 671 & 60 & 11.2 & & \\
\hline
\end{tabular}

\begin{tabular}{|l|l|l|l|l|l|}
\hline Anova Cubes & Sum of Squares & df & Mean Square & $\mathrm{F}$ & $\mathrm{p}$ \\
\hline PCS & 159 & 2 & 79.4 & 6.21 & 0.004 \\
\hline Residuals & 671 & 60 & 11.2 & & \\
\hline
\end{tabular}

\begin{tabular}{|l|l|l|l|l|l|}
\hline Anova Matrices & Sum of Squares & df & Mean Square & F & p \\
\hline PCS & 71.1 & 2 & 35.54 & 5.52 & 0.006 \\
\hline Residuals & 671 & 60 & 11.2 & & \\
\hline
\end{tabular}

\begin{tabular}{|l|l|l|l|l|l|}
\hline Anova Mém. chiffres & Sum of Squares & df & Mean Square & F & p \\
\hline PCS & 31.7 & 2 & 15.86 & 2.42 & 0.098 \\
\hline Residuals & 393.1 & 60 & 6.55 & & \\
\hline
\end{tabular}

\begin{tabular}{|l|l|l|l|l|l|}
\hline Anova Code & Sum of Squares & df & Mean Square & F & p \\
\hline PCS & 18.7 & 2 & 9.35 & 1.09 & 0.34301 \\
\hline Residuals & 514.4 & 60 & 8.57 & & \\
\hline
\end{tabular}

\begin{tabular}{|l|l|l|l|l|l|}
\hline Anova Symboles & Sum of Squares & df & Mean Square & F & p \\
\hline PCS & 19.1 & 2 & 9.54 & 0.960 & 0.389 \\
\hline Residuals & 596.0 & 60 & 9.93 & & \\
\hline
\end{tabular}

Références : The jamovi project (2020). Jamovi. (Version) [1.2Computer Software]. Retrieved from https://www.jamovi.org. 


\section{définitionnelles, à caractériser différents contenus sémantiques et à identifier la façon} dont les enfants combinent ces différentes informations

A trois types d'énoncés (catégoriels : $C$, fonctionnels : $F$, perceptifs : $P$ ) correspondent 15 combinaisons de réponses possibles. Les patterns de réponses sont codés selon les initiales $\mathrm{C}=$ Catégoriel $; \mathrm{F}=$ Fonctionnel ; $\mathrm{P}=$ Perceptif. Par exemple, un pattern C-P-F signifie que la définition est constituée d'un énoncé catégoriel puis d'un énoncé perceptif, puis d'un énoncé fonctionnel. Un codage simple F signifie qu'une seule information, de nature fonctionnelle, a été fournie dans la définition.

Tableau 2 : Répartition des définitions en fonction de leurs schémas de codage par classe sociale

\begin{tabular}{|l|l|l|l|l|l|l|l|l|l|l|l|l|l|l|l|}
\hline Patterns de réponses & C & CF & CP & CFP & CPF & F & FC & FP & FCP & FPC & P & PC & PF & PCF & PFC \\
\hline Classes défavorisées & 18 & 16 & 16 & 4 & 6 & 29 & 4 & 19 & 0 & 1 & 20 & 2 & 13 & 0 & 0 \\
\hline Classes moyennes & 19 & 15 & 13 & 6 & 10 & 36 & 7 & 18 & 1 & 1 & 17 & 0 & 11 & 0 & 6 \\
\hline
\end{tabular}

Corela, 19-1 | 2021 


\begin{tabular}{|l|l|l|l|l|l|l|l|l|l|l|l|l|l|l|l|}
\hline Classes favorisées & 25 & 20 & 25 & 12 & 12 & 33 & 0 & 8 & 0 & 0 & 14 & 2 & 11 & 0 & 4 \\
\hline Totaux & 62 & 51 & 54 & 22 & 28 & 98 & 11 & 45 & 1 & 2 & 51 & 4 & 35 & 0 & 10 \\
\hline
\end{tabular}

Figure 1 : Histogramme de répartition des définitions en fonction de leurs schémas de codage par classe sociale (version 15 patterns)

Pour l'ensemble de l'échantillon et sur l'ensemble des neuf mots, les réponses les plus fréquentes sont celles où les enfants n'utilisent qu'un seul type de codage définitionnel : soit catégoriel, soit fonctionnel, soit perceptif. Ces codages simples représentent $44,5 \%$ de la totalité des définitions produites. Dans ces cas de codage simple, les sujets marquent une préférence pour les informations fonctionnelles du type «c'est pour...; ça sert à...", comparativement aux deux autres types de codage: plus de $20,7 \%$ des définitions produites sont uniquement sur le registre fonctionnel tandis que $13,1 \%$ sont seulement sur le registre catégoriel et 10,7\% exclusivement sur le registre perceptif. Tout se passe comme si, pour beaucoup d'enfants à cet âge encore, l'énoncé d'une seule composante du mot, préférentiellement fonctionnelle, suffisait à en saisir toutes les facettes.

Certains patterns sont très rarement produits, voire totalement absents des réponses des enfants : il s'agit des patterns P-C-F (par ex.: " une vache: ça a des cornes, c'est un animal, et ça produit du lait »), F-P-C (par ex. : « un nénuphar c'est pour les grenouilles, c'est sur l'eau et c'est une plante »), FPC (par ex. : « une bicyclette c'est pour se promener, ça a deux roues et c'est un véhicule », FCP (par ex. : « une horloge ça donne l'heure, c'est un objet avec des aiguilles») . Les types de codages définitionnels produits par les enfants ne sont donc pas aléatoires. Lorsqu'il y a codage multiple, il apparaît que certaines combinaisons sont plus acceptables que d'autres. Les enfants, à cet âge, semblent commencer à faire fonctionner un certain nombre de règles implicites : par exemple, qu'une définition de nom doit aller du général (la catégorie générique) vers le particulier (les traits d'apparence) plutôt que l'inverse ; ou bien qu'une définition ne se termine pas par le terme générique... La catégorie supra-ordonnée, lorsqu'elle est énoncée, l'est le plus souvent en première position, très rarement en deuxième ou troisième place dans le schéma de codage définitionnel. Les combinaisons du type " $\mathrm{X}$ est un $\mathrm{Y}$ qui..." apparaissent fréquemment (Ex. : une vache est un animal qui mange de l'herbe (C-P); une vache est un animal qui donne du lait (C-F). Un nénuphar est une plante avec une feuille verte (C-P) ; un nénuphar est une plante d'eau pour que les grenouilles sautent dessus (CF).

Compte-tenu de la faible fréquence de certains patterns de codages définitionnels, nous avons pu procéder aux regroupements suivants afin de faciliter l'analyse et la lecture des résultats :

$$
\mathbf{C F}=[\mathrm{CF}+\mathrm{CFP}]-\mathbf{C P}=[\mathrm{CP}+\mathrm{CPF}]-\mathbf{F}+=[\mathrm{FP}+\mathrm{FC}+\mathrm{FCP}+\mathrm{FPC}]-\mathbf{P}+=[\mathrm{PF}+\mathrm{PC}+\mathrm{PFC}+\mathrm{PCF}]
$$

Tableau 3 : Répartition des définitions en fonction de leurs schémas de codage par classe sociale (version 7 patterns)

\begin{tabular}{|l|l|l|l|l|l|l|l|}
\hline Patterns de réponses & C & CF & CP & F & F+ & P & P+ \\
\hline Classes défavorisées & 18 & 20 & 22 & 29 & 24 & 20 & 15 \\
\hline Classes moyennes & 19 & 21 & 23 & 36 & 27 & 17 & 17 \\
\hline
\end{tabular}




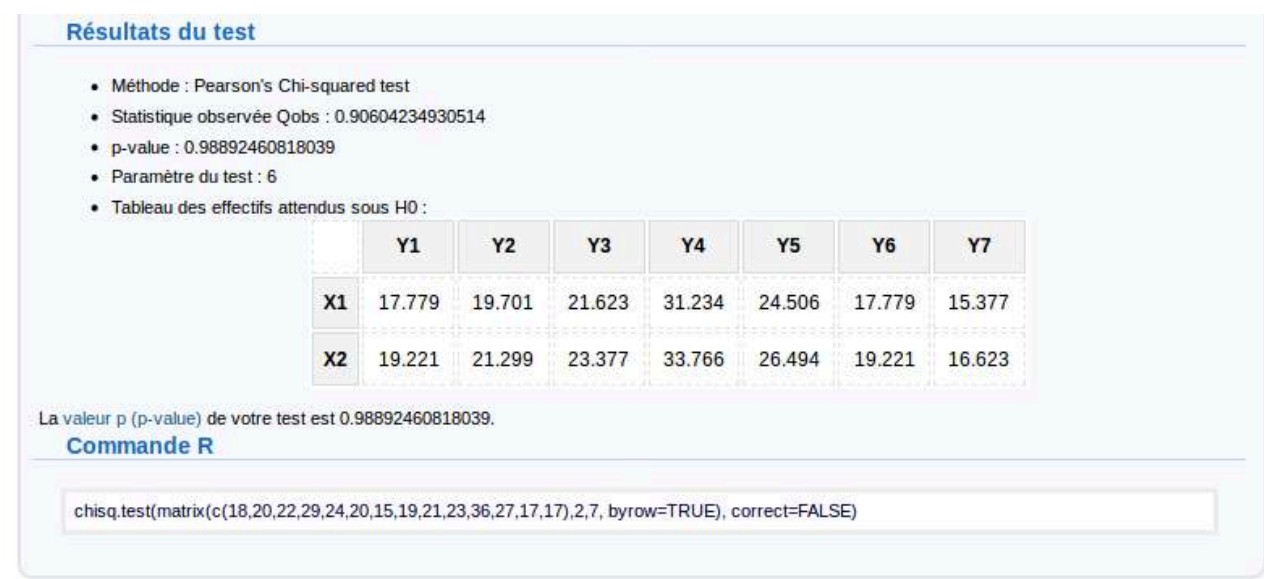

- Classes moyennes / classes favorisées : chi2 $(6)=16,99, \mathrm{p}=0,009=>\mathrm{H} 0$ rejetée : il existe une différence significative de codage définitionnel entre classes moyennes et classes favorisées. Rèsultats du test

- Methode : Pearson's Chi-squared test

- Statistique observèe Qobs : 16.998238976769

- p-value : 0.009289718535464

- Paramètre du test : 6

- Tableau des effectifs attendus sous HO

\begin{tabular}{|l|c|c|c|c|c|c|c|}
\hline & Y1 & Y2 & Y3 & Y4 & Y5 & Y6 & Y7 \\
\hline $\mathbf{X 1}$ & 21.595 & 26.012 & 29.448 & 33.865 & 17.178 & 15.215 & 16.687 \\
\hline $\mathbf{X 2}$ & 22.405 & 26.988 & 30.552 & 35.135 & 17.822 & 15.785 & 17.313 \\
\hline
\end{tabular}

La valeur $p$ (p-value) de votre test est 0.009289718535464 . Commande $\mathrm{R}$

chisq.test(matrix(c(19,21,23,36,27,17,17,25,32,37,33,8,14,17),2,7, byrow=TRUE), correct=FALSE)

- Classes défavorisées/ classes favorisées : chi2 (6) =16,18, p=0,012 =>H0 rejetée : il existe une différence significative de codage définitionnel entre classes défavorisées et classes favorisées.

Résultats du test

- Méthode : Pearson's Chi-squared test

- Statistique observèe Qobs : 16.185553840075

- p-value : 0.012791928089071

- Parametre du test : 6

- Tableau des effectifs attendus sous HO :

\begin{tabular}{|c|c|c|c|c|c|c|c|}
\hline & Y1 & Y2 & Y3 & Y4 & Y5 & Y6 & Y7 \\
\hline X1 & 20.268 & 24.51 & 27.809 & 29.223 & 15.083 & 16.025 & 15.083 \\
\hline X2 & 22.732 & 27.49 & 31.191 & 32.777 & 16.917 & 17.975 & 16.917 \\
\hline
\end{tabular}

La valeur $p$ ( $p$-value) de votre test est 0.012791928089071 Commande R

chisq.test(matrix(c(18,20,22,29,24,20,15,25,32,37,33,8,14,17), 2,7, byrow=TRUE), correct=FALSE)

En ce qui concerne l'hypothèse selon laquelle il existerait des préférences différenciées pour certains patterns de réponses selon la catégorie sociale d'appartenance, nos résultats conduisent à penser que les enfants issus de milieux sociaux favorisés se distinguent des catégories sociales défavorisées $(\mathrm{p}=0,012)$ et des catégories sociales moyennes $(p=0,009)$ en privilégiant davantage les schémas $C$, $C F$ et $C P$. Dans le même temps, les enfants des milieux favorisés tendent à moins mobiliser les combinaisons $\mathrm{F}+$ que ne le font les enfants des deux autres milieux socio-économiques. En d'autres 
termes, les enfants issus des milieux aisés tendent un peu plus que les autres enfants à produire des codages définitionnels qui s'organisent à partir du terme désignant la catégorie générique, ce qui indiquerait une évolution un peu plus rapide que les classes défavorisées et moyennes vers des schémas de codages plus matures. Nos résultats suggèrent qu'il n'y a pas de différence entre la catégorie défavorisée et la catégorie moyenne $(p=0,989)$ quant aux schémas de codage définitionnel mobilisés. Les enfants issus de ces deux catégories sociales semblent évoluer au même rythme, plus lent que ceux de milieux favorisés vers la forme définitionnelle canonique, avec un ancrage encore important sur la dimension fonctionnelle en cas d'énoncés multiples. Mais ces effets socio-différentiels n'apparaissent pas pour les définitions qui ne proposent qu'un seul type de contenu (P, F ou C) : il n'y a pas d'effet de la catégorie sociale d'origine pour les codages simples.

51 Afin d'affiner les analyses des mécanismes de construction de définitions, nous allons observer à présent ce qu'il en est de la première information produite par les enfants.

\subsection{Nature du premier codage définitionnel}

52 La première information proposée par l'enfant dans sa définition est aussi la plus accessible pour lui. C'est à partir de ce premier élément énoncé que va se structurer la suite de la définition.

Tableau 4 : Fréquence et pourcentage du premier codage définitionnel

\begin{tabular}{|l|l|l|l|l|}
\hline Effectifs de 1er codage & Classe défavorisée & Classe moyenne & Classe favorisée & Total \\
\hline Catégoriel & 60 & 63 & 94 & 217 \\
\hline Fonctionnel & 53 & 63 & 41 & 157 \\
\hline Perceptif & 35 & 34 & 31 & 100 \\
\hline \% de de 1 $1^{\text {er codage }}$ & Classe défavorisée & Classe moyenne & Classe favorisée & Total \\
\hline Catégoriel & $40,5 \%$ & $39,40 \%$ & $56,60 \%$ & $45,80 \%$ \\
\hline Fonctionnel & $35,80 \%$ & $39,40 \%$ & $24,70 \%$ & $33,10 \%$ \\
\hline Perceptif & $23,60 \%$ & $21,20 \%$ & $18,70 \%$ & $21,10 \%$ \\
\hline
\end{tabular}

53 Les chi-2 réalisés ont permis d'examiner l'hypothèse d'une relation entre classe sociale et premier codage définitionnel :

- classes défavorisées/classes moyennes/classes favorisées : chi-2 (4) =13,275, $p=0.01$

- classes défavorisées/classes moyennes : chi-2(2) =0,48, p=0,785

- classes moyennes/classes favorisées : $\operatorname{chi}-2(2)=10,80, p=0,004$

- classes défavorisées/classes favorisées : $\operatorname{chi}-2(2)=8,27, p=0,016$

Chi-2 pour le premier codage définitionnel pour les 3 classes sociales

$\mathrm{X} 1=$ classe défavorisée, $\mathrm{X} 2=$ classe moyenne, $\mathrm{X} 3=$ classe favorisée 
$\mathrm{Y} 1=$ codage par la catégorie, $\mathrm{Y} 2=$ codage par la fonction, $\mathrm{Y} 3=$ codage par la perception

HO : la classe sociale n'influence pas le premier codage définitionnel

Classes défavorisées/classes moyennes/classes favorisées: chi-2 (4) =13,275, p=0.01 => H0 rejetée : la classe sociale influence le premier codage définitionnel

Résultats du test

- Methode : Pearson's Chi-squared tes

Statistique observée Qobs : 13.2749313935

p-value : 0.01000770619054

- Paramétre du test :4

- Tableau des effectifs attendus sous $\mathrm{HO}$ :

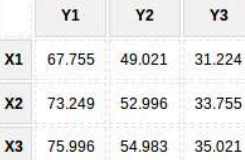

La valeur $p$ (p-value) de votre test est 0.010007706190545

Commande R

chisq.test(mattix(c(60,53,35, 63,63,34,94,41,31),3,3, byrow=TRUE), correct=FALSE)

classe favorisée (X2)

- classes moyennes/classes favorisées: $\operatorname{chi}-2(2)=10,80, p=0,004=>$ H0 rejetée. Le premier codage n'est pas identique selon la classe moyenne et la classe favorisée.

Rèsultats du test

Methode : Pearson's Chi-squared test

- Statistique observée Qobs : 10.806557973073

- p-value : 0.0045017953885745

- Parametre du test : 2

- Tableau des effectifs attendus sous HO:

de votre test est 0.78547509413738 .

\begin{tabular}{|c|c|c|c|}
\hline & Y1 & Y2 & Y3 \\
\hline X1 & 77.055 & 51.043 & 31.902 \\
\hline X2 & 79.945 & 52.957 & 33.098 \\
\hline
\end{tabular}

La valeur $p$ (p-value) de votre test est 0.0045017953885745 .

Commande $\mathrm{R}$

chisq.test(matrix (c( $63,63,34,94,41,31), 2,3$, byrow=TRUE), correct=FALSE) 
H0 : le premier codage est identique entre classe défavorisée (X1) et classe favorisée (X2)

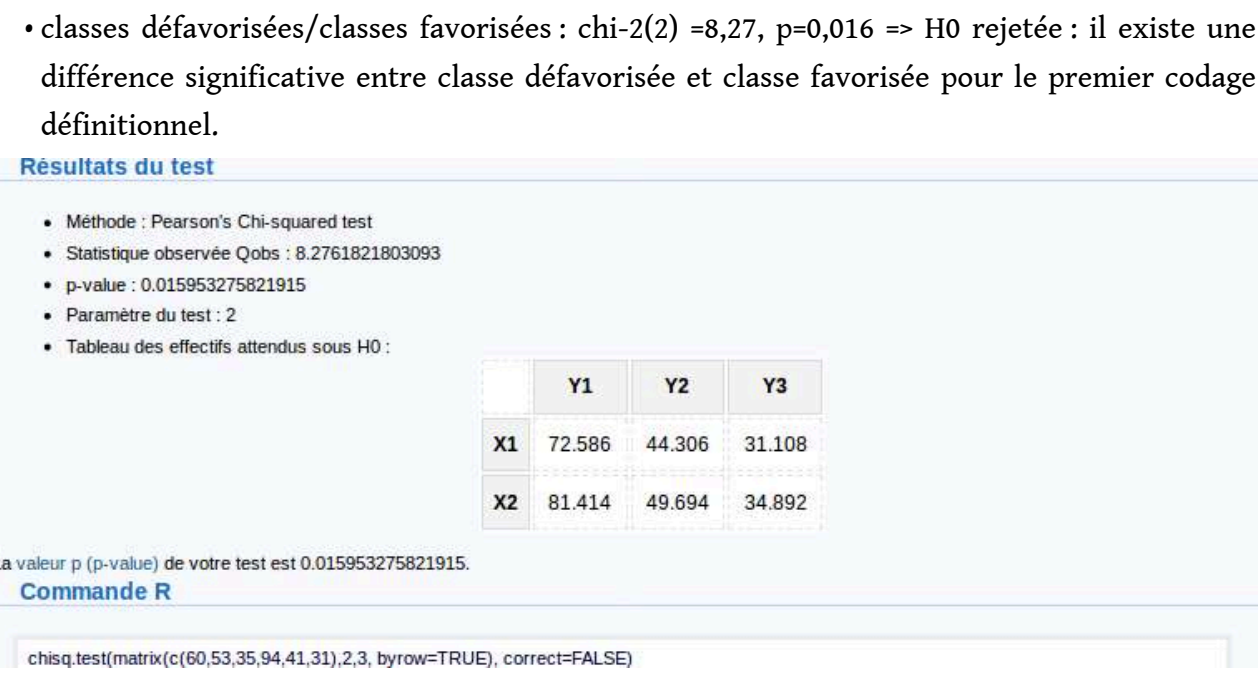

Ces résultats suggèrent que la classe sociale d'origine des enfants joue un rôle quant à l'accessibilité du premier codage définitionnel $(\mathrm{p}=0,01)$. Les enfants issus de la classe favorisée se distinguent des deux autres catégories sociales quant au type d'information mobilisée en premier : ils font plus fréquemment appel à des énoncés catégoriels $(56,6 \%$ contre respectivement $40,5 \%$ et $39,4 \%$ pour les enfants des classes défavorisées $(\mathrm{p}=0,016)$ et moyennes $(\mathrm{p}=0,004)$ et moins souvent appel à des énoncés fonctionnels $(24,7 \%$ contre respectivement $35,8 \%$ et $39,4 \%$ pour les catégories défavorisées et moyennes). Il n'y a pas de différences quant aux pourcentages de définitions privilégiant une information de type perceptif $(23,6 \% ; 21,2 \%$ et $18,7 \%)$. L'analyse au moyen de tests multiples ne relèvent pas de différences entre les catégories sociales défavorisées et moyennes $(p=0,785)$. Seule la classe favorisée se distingue des deux autres sur la nature du premier codage définitionnel.

\section{Discussion}

Cette recherche, rappelons-le, visait à identifier le comportement définitionnel d'enfants de 9-11 ans puis à examiner d'éventuelles variations en fonction de leur appartenance sociale.

Les études développementales (Benelli \& al., 1988, Nelson, 1978, Wehren \& al., 1981) avaient montré qu'il existe des schémas de réponses préférentiels qui évoluent avec l'âge vers la forme canonique. Cette recherche confirme que c'est autour de 9-11 ans que les enfants commencent à structurer leurs définitions comme le font les adultes (voir aussi Marinellie, 2010, Kurland \& al., 1997, Kikas, 1993, Wehren, 1981). Ces recherches développementales ne relèvent que peu de différences de maturité des définitions entre 9 et 11 ans. Le recueil de nouvelles données sur un échantillon plus important pourrait nous permettre d'examiner plus précisément ce point.

Même si près de la moitié des 567 définitions transcrites et analysées ici restent encore, $\mathrm{du}$ point de vue de la signification, très axées sur la fonction des objets, nos résultats vont dans le sens d'une maturation des définitions vers la forme canonique (Nippold, 
1995, Ehrlich \& al., 1978), en lien avec l'acquisition des capacités de conceptualisation (Kikas, 1993, Gandia, 2016) qui se manifestent chez les enfants de 9-11 ans.

Il est vraisemblable que les schémas de réponses produits soient sous l'influence de facteurs sémantiques, comme la nature des noms (objets versus êtres vivants...), ou liés à des contraintes de structure de la langue (par exemple dans le mot parapluie il y a aussi le mot pluie). Nous postulons avec Snow $(1990,2017)$ que cet apprentissage plus ou moins incident de la forme définitionnelle normative, s'opère à l'école pour l'essentiel.

Le principal apport de cette étude concerne le rôle de la classe sociale d'origine sur les aspects structurels et normatifs des définitions orales émises par les enfants. Qu'il s'agisse des schémas de réponses ou de l'accessibilité de la première information (qui va orienter la suite de la définition) des différences significatives sont observées entre la classe favorisée et les deux autres catégories étudiées. Une analyse plus fine indique que ces différences n'apparaissent pas pour les définitions à codage unidimensionnel, qui concernent la moitié de l'échantillon. L'absence de différences entre catégories socialement défavorisées et classes moyennes constitue un résultat qui peut laisser penser que le milieu social d'origine aurait un rôle modéré sur l'organisation des définitions. Si nos résultats tendent à montrer une évolution plus rapide des enfants issus des milieux socio-économiques favorisés vers la forme définitionnelle canonique, cet effet socio-différentiel ne semble pas aussi marquée qu'il ne l'est si l'on ne considère que la performance globale à l'épreuve de vocabulaire de l'échelle de Weschler (voir aussi Mercy \& al., 1982, Grégoire, 2007). Rappelons que cette épreuve standardisée produit un score à partir d'un nombre de mots correctement définis mais ne tient pas compte de l'organisation des énoncés. Ainsi, il semblerait que le rôle de la classe sociale sur les aspects formels des définitions soit somme toute assez modeste comparativement aux différences de performances mesurées au test standardisé entre favorisés et défavorisés.

Nos travaux soulignent cette tension entre contenu et forme définitionnelle. Ils invitent à interroger l'enseignement-apprentissage de la compréhension lexicale dans une perspective de lutte contre les inégalités scolaires. Qu'enseigne-t-on? Qu'évalue-ton? Pour quels publics?

L'école semble avoir du mal à compenser les inégalités linguistiques (Brunner \& al., 2019). Mais si elle peine à influer sur les différences entre enfants quant au nombre de mots de vocabulaire entendus depuis la naissance (Becker, 2011, Hart \& al., 1995), aurait-elle un rôle plus efficace sur le lexique profond, notamment par des actions qui faciliteraient l'accès à la compréhension des mots?

\section{BIBLIOGRAPHY}

Al-Issa, I. (1969). The Development of Word Definition in Children. The Journal of Genetic Psychology, 114(1), 25-28. https://doi.org/10.1080/00221325.1969.10533835 
Anglin, J. (1970). The growth of word meaning. Cambridge: MIT Press.

Becker, B. (2011). Social disparities in children's vocabulary in early childhood. Does pre-school education help to close the gap?1: Social disparities in children's vocabulary in early childhood. The British Journal of Sociology, 62(1), 69-88. https://doi.org/10.1111/j.1468-4446.2010.01345.x

Benelli, B., Arcuri, L., \& Marchesini, G. (1988). Cognitive and linguistic factors in the development of word definitions. Journal of Child Language, 15, 619-635. https://doi.org/10.1017/ S0305000900012599

Benelli, B., Belacchi, C., Gini, G., \& Lucangeli, D. (2006). 'To define means to say what you know about things': the development of definitional skills as metalinguistic acquisition. Journal of Child Language, 33(01), 71. https://doi.org/10.1017/\$0305000905007312

Brunner, A., Maurin, L. (Dir.) (2019). Rapport sur les inégalités en France. Tours. Observatoire des inégalités (Ed).

Cravatte, A. (1980). Comment les enfants expliquent-ils les mots? In : Langages, 14e année, n59. Conduites langagières et sociolinguistiques scolaire. pp. 87-96 https://doi.org/10.3406/lgge.

1980.1857

Croizet, J.-C., \& Claire, T. (1998). Extending the concept of stereotype and threat to social class: The intellectual underperformance of students from low socioeconimic backgrounds. Personality and Social Psychology Bulletin, 24(6), 588-594. https://doi.org/10.1177/0146167298246003

Ehrlich, S., Bramaud Du Boucheron, G., Florin, A. (1978). Le développement des compétences lexicales à l'école Primaire. PUF. http://pascal-francis.inist.fr/vibad/index.php? action=getRecordDetail\&idt=PASCAL7850311025

Fernald, A., Weisleder, A. (2015). Twenty Years after "Meaningful Differences," It's Time to Reframe the "Deficit" Debate about the Importance of Children's Early Language Experience. Human Development, 58:1-4. https://doi: 10.1159/000375515

Goudeau, S., Autin, F., \& Croizet, J. C. (2017). Etudier, mesurer et manipuler la classe sociale en psychologie sociale : Approches economiques, symboliques et culturelles [Studying, measuring and manipulating social class in social psychology: Economic, symbolic and cultural approaches]. International Review of Social Psychology, 30(1), Article 1-19.

Gandía, A. M. (2016). On Word Definition in Children and Adults: Effects of Word Category and Level of Abstraction. http://diposit.ub.edu/dspace/handle/2445/107763

Grégoire, J. (2007). L'examen clinique de l'intelligence de l'enfant : Fondements et pratique du Wisc-IV. Mardaga.

Grobon S, Panico L, Solaz A., (2019). Inégalités socioéconomiques dans le développement langagier et moteur des enfants à 2 ans. Bull Epidémiol Hebd. 2019;(1):2-9. http:// invs.santepubliquefrance.fr/beh/2019/1/2019_1_1.html

Hadley, E. B., Dickinson, D. K., Hirsh-Pasek, K., Michnick Golinkoff, R.(2019). Building Semantic Networks: The Impact of a Vocabulary Intervention on Preschoolers' Depth of Word Knowledge. Reading Research Quarterly, Volume 54, Issue , 41-61. https://doi.org/10.1002/rrq.225

Hart, B., \& Risley, T. R. (1995). Meaningful differences in the everyday experience of young American children. Baltimore, MD, US: Paul H Brookes Publishing.

Hoff, E. (2003). The Specificity of Environmental Influence: Socioeconomic Status Affects Early Vocabulary Development Via Maternal Speech. Child Development, 74(5), 1368-1378. https:// doi.org/10.1111/1467-8624.00612 
Kikas, E. (1993). The Development of Word Definitions in Children. Journal of Russian \& East European Psychology, 31(2), 40-54. https://doi.org/10.2753/RPO1061-0405310240

Kraus, M. W., \& Stephens, N. M. (2012). A road map for an emerging psychology of social class. Social and Personality Psychology Compass, 6(9), 642-656._https://doi.org/10.1111/j. 1751-9004.2012.00453.x

Kurland, B. F. \& Snow, C. (1997). Longitudinal measurement of growth in definitional skill. Journal of Child Language 24, 603-26. https://doi.org/10.1017/S0305000997003243

Lahey, M. (1988). Language disorders and language development. Needham, MA: Macmillan.

Marinellie, S. A. (2010). The Understanding of Word Definitions in School-Age Children. Journal of Psycholinguistic Research, 39(3),179-197. https://doi.org/10.1007/s10936-009-9132-4

McGhee-Bidlack, B. (1991). The development of noun definitions: a metalinguistic analysis. Journal of Child Language 18, 417-34. https://doi.org/10.1017/S0305000900011132

Mercy, J.A. \& Steelman, L.C. (1982). Familial influence on the intellectual attainment of children. American Sociological Review, 47, 532-542.

Nippold, M. A. (1995). School-Age Children and Adolescents. Language Speech and Hearing Services in Schools, 26(4), 320. https:// doi:10.1044/0161-1461.2604.320

Ouellette, G. P. (2006). What's meaning got to do with it: The role of vocabulary in word reading and reading comprehension. Journal of Educational Psychology, 98(3), 554-566. https://doi.org/ 10.1037/0022-0663.98.3.554

Pan, B.A. (2012). Assessing Vocabulary Skills. In E. Hoff (Éd.), Research Methods in Child Language (p. 100-112). https://doi.org/10.1002/9781444344035.ch7

Salazar Orvig, A., Préneron, C., \& Kugler, M.-P. (1988). Les conduites de définition chez des enfants non-lecteurs. Langue française, 80(1), 83-97. https://doi.org/10.3406/lfr.1988.4762

Simoes-Loureiro, I. \& Lefebvre, L. (2015). Développement d'un questionnaire de connaissances sémantiques des objets naturels et manufacturés pour enfants de 5 à 9 ans. L'Année psychologique, vol. 115(3), 409-434. https://doi:10.4074/S0003503315000172.

Snow, C. E. (1990). The development of definitional skill. Journal of Child Language 17,697-710. https://doi.org/10.1017/S0305000900010953

Snow, C. E., Cancino, H., Temple, J. D., \& Schley, S. (1991). Giving formal definitions: A linguistic or metalinguistic skill? In E. Bialystok (Éd.), Language processing in bilingual children (p. 90-112). https://doi.org/10.1017/CBO9780511620652.007

Snow, C. E. (2017) The role of vocabulary versus knowledge in children's language learning: a fifty-year perspective / El papel del vocabulario frente al conocimiento en el aprendizaje lingüístico de los niños: una perspectiva de cincuenta años, Infancia y Aprendizaje, 40:1, 1-18, https://doi.org/10.1080/02103702.2016.1263449

Watson, R. (1985). Towards a theory of definition. Journal of Child Language, 12(01). https:// doi.org/10.1017/S0305000900006309

Wechsler, D. (2005). WISC-IV. Echelle d'intelligence de Wechsler pour enfants et adolescents. Quatrième édition. Paris : ECPA.

Wehren, A., De Lisi, R., \& Arnold, M. (1981). The development of noun definitions. Journal of Child Language, 8, 165-175. https://doi.org/10.1017/\$0305000900003081 


\section{APPENDIXES}

Annexe $1:$ Tableaux et graphiques

Tableau 1 : Note standard moyenne à 6 épreuves du WISC-4 par catégorie sociale

\begin{tabular}{|l|l|l|l|}
\hline & Classe défavorisée & Classe moyenne & Classe favorisée \\
\hline $\begin{array}{l}\text { Vocabulaire } \\
\text { ANOVA-(2) }=17,2, \mathrm{p}<.001\end{array}$ & 6,14 & 8,14 & 12,10 \\
\hline $\begin{array}{l}\text { Cubes } \\
\text { ANOVA-(2) = 6,21, p=0.004 }\end{array}$ & 7,14 & 7,38 & 10,24 \\
\hline $\begin{array}{l}\text { Matrices } \\
\text { ANOVA-(2) }=5,52, \mathrm{p}=0.006\end{array}$ & 8,14 & 8,81 & 10,62 \\
\hline $\begin{array}{l}\text { Mém. des chiffres } \\
\text { ANOVA-(2) }=2,42, \mathrm{p}=0.098\end{array}$ & 9,14 & 7,86 & 9,29 \\
\hline $\begin{array}{l}\text { Code } \\
\text { ANOVA-(2) =1,09, p=0.343 }\end{array}$ & 10,14 & 9,14 & 8,81 \\
\hline $\begin{array}{l}\text { Symboles } \\
\text { ANOVA-(2) }=0,960, \mathrm{p}=0.389\end{array}$ & 11,14 & 9,24 & 10,38 \\
\hline
\end{tabular}

Tableau 2 : Répartition des définitions en fonction de leurs schémas de codage par classe sociale

\begin{tabular}{|l|l|l|l|l|l|l|l|l|l|l|l|l|l|l|l|}
\hline Patterns de réponses & C & CF & CP & CFP & CPF & F & FC & FP & FCP & FPC & P & PC & PF & PCF & PFC \\
\hline Classes défavorisées & 18 & 16 & 16 & 4 & 6 & 29 & 4 & 19 & 0 & 1 & 20 & 2 & 13 & 0 & 0 \\
\hline Classes moyennes & 19 & 15 & 13 & 6 & 10 & 36 & 7 & 18 & 1 & 1 & 17 & 0 & 11 & 0 & 6 \\
\hline Classes favorisées & 25 & 20 & 25 & 12 & 12 & 33 & 0 & 8 & 0 & 0 & 14 & 2 & 11 & 0 & 4 \\
\hline Totaux & 62 & 51 & 54 & 22 & 28 & 98 & 11 & 45 & 1 & 2 & 51 & 4 & 35 & 0 & 10 \\
\hline
\end{tabular}

Figure 1 : Histogramme de répartition des définitions en fonction de leurs schémas de codage par classe sociale (version 15 patterns)

Tableau 3 : Répartition des définitions en fonction de leurs schémas de codage par classe sociale (version 7 patterns)

\begin{tabular}{|l|l|l|l|l|l|l|l|}
\hline Patterns de réponses & C & CF & CP & F & F+ & P & P+ \\
\hline
\end{tabular}




\begin{tabular}{|l|l|l|l|l|l|l|l|}
\hline Classes défavorisées & 18 & 20 & 22 & 29 & 24 & 20 & 15 \\
\hline Classes moyennes & 19 & 21 & 23 & 36 & 27 & 17 & 17 \\
\hline Classes favorisées & 25 & 32 & 37 & 33 & 8 & 14 & 17 \\
\hline Total & 62 & 73 & 82 & 98 & 59 & 51 & 49 \\
\hline \% de patterns & $\mathbf{C}$ & $\mathbf{C F}$ & $\mathbf{C P}$ & $\mathbf{F}$ & $\mathbf{F}+$ & $\mathbf{P}$ & $\mathbf{P}+$ \\
\hline Classes défavorisées & $29,03 \%$ & $27,40 \%$ & $26,83 \%$ & $29,59 \%$ & $40,68 \%$ & $39,22 \%$ & $30,61 \%$ \\
\hline Classes moyennes & $30,65 \%$ & $28,77 \%$ & $28,05 \%$ & $36,73 \%$ & $45,76 \%$ & $33,33 \%$ & $34,69 \%$ \\
\hline Classes favorisées & $40,32 \%$ & $43,83 \%$ & $45,12 \%$ & $33,67 \%$ & $13,56 \%$ & $27,45 \%$ & $34,69 \%$ \\
\hline \% Total & $13,08 \%$ & $15,40 \%$ & $17,30 \%$ & $20,68 \%$ & $12,45 \%$ & $10,76 \%$ & $10,34 \%$ \\
\hline
\end{tabular}

Tableau 4 : Fréquence et pourcentage du premier codage définitionnel

\begin{tabular}{|c|c|c|c|c|}
\hline Effectifs de 1er codage & Classe défavorisée & Classe moyenne & Classe favorisée & Total \\
\hline Catégoriel & 60 & 63 & 94 & 217 \\
\hline Fonctionnel & 53 & 63 & 41 & 157 \\
\hline Perceptif & 35 & 34 & 31 & 100 \\
\hline$\%$ de de $1^{\mathrm{er}}$ codage & Classe défavorisée & Classe moyenne & Classe favorisée & Total \\
\hline Catégoriel & $40,5 \%$ & $39,40 \%$ & $56,60 \%$ & $45,80 \%$ \\
\hline Fonctionnel & $35,80 \%$ & $39,40 \%$ & $24,70 \%$ & $33,10 \%$ \\
\hline Perceptif & $23,60 \%$ & $21,20 \%$ & $18,70 \%$ & $21,10 \%$ \\
\hline
\end{tabular}

Annexe 2. Codage de la classe sociale

Nous avons mesuré la classe sociale des élèves à partir des professions et catégories socio-professionnelles des parents, communiquées par les établissements scolaires et confirmées lors des entretiens parentaux. Ces différentes professions et catégories socioprofessionnelles (PCS) sont relatives à la nomenclature statistique de l'Institut national de la statistique et des études économiques (Insee). Cette nomenclature, créée en 1982, comprend 486 professions regroupées en 31 catégories socioprofessionnelles. Nous avons effectué une catégorisation selon trois classes sociales, sur la base suivante :

Classes populaires :

Agriculteurs exploitants

Artisans, commerçants et assimilés 
Employés civils et agents de service de la fonction publique

Employés administratifs d'entreprise (sauf informaticiens)

Employés de commerce

Personnels des services directs aux particuliers

Ouvriers qualifiés, ouvriers non qualifiés, ouvriers agricoles

Chômeurs n'ayant jamais travaillé

Inactifs divers (autres que retraités)

Classes moyennes :

Professions intermédiaires de la santé et du travail social

Clergé, religieux

Professions intermédiaires administratives de la fonction publique

Professions intermédiaires administratives et commerciales des entreprises

Techniciens

Contremaîtres, agents de maîtrise

Policiers et militaires

Classes favorisées :

Chefs d'entreprise de 10 salariés ou plus

Professions libérales

Cadres de la fonction publique

Professeurs, professions scientifiques

Professions de l'information, des arts et des spectacles

Cadres administratifs et commerciaux d'entreprise

Ingénieurs et cadres techniques d'entreprise

Professeurs des écoles, instituteurs et assimilés

Pour notre étude comme pour la plupart des recherches actuelles, les professeurs des écoles ont été classés dans la catégorie favorisée (Goudeau \& al., 2017). La nomenclature INSEE-PCS de 2017 avait maintenu cette profession dans la catégorie 3 des professions intermédiaires, sans tenir compte de leur alignement statutaire et salarial sur les professeurs certifiés du second degré (voir aussi les préconisations du rapport du Conseil National de l'Information Statistique ainsi que celles de la DEPP).

«Le classement de ceux-ci dans les professions intermédiaires pose en effet question alors que le recrutement de l'ensemble des professeurs du primaire et du secondaire est, depuis 2011, réalisé à un niveau bac +5 , et que la grille de rémunération du corps des professeurs des écoles est alignée sur celle des professeurs certifiés (classés parmi les cadres et professions intellectuelles supérieures)

Conseil national de l'information statistique. La nomenclature des PCS et ses usages. Etat des lieux et défis. Octobre 2018 pp. 38 


\section{NOTES}

1. Les notes standard varient de 1 à 19 . Entre 7 et 13, une note standard indique une performance dans les variations de la norme. En dessous de 7, la note indique une performance très inférieure à la norme. Au-dessus de 13, la performance est jugée très au-dessus de la norme.

\section{ABSTRACTS}

This study analyzes the oral responses of 63 french students aged 9-11 years old on a vocabulary task such as word definition. The overall performance, but also the organization and accessibility of categorical, functional or perceptual information are examined by considering the children's social background. The quantitative results of the study confirm those obtained from research on the relationship between socio-economic background and vocabulary breadth. The qualitative data from the analysis of the structure of 567 definitions, highlight moderate differences in the evolution towards the canonical form of the definition, according to social class. This questions our ways of thinking about the teaching-learning of vocabulary and invites us to further explore the mechanisms at play in the unequal access to knowledge of the meaning of words.

Cette étude analyse les réponses orales de 63 élèves français de 9-11 ans lors d'une tâche de vocabulaire du type définition de mots. La performance globale, mais aussi l'organisation et l'accessibilité des informations catégorielles, fonctionnelles ou perceptives sont examinées en considérant l'origine sociale des enfants. Les résultats quantitatifs de l'étude confirment ceux obtenus par les recherches sur la relation entre milieu socio-économique et étendue du vocabulaire. Les données qualitatives, tirées de l'analyse de la structure de 567 définitions, mettent en évidence des différences modérées de l'évolution vers la forme canonique de la définition, selon la classe sociale. Ceci interroge nos manières de penser l'enseignementapprentissage du vocabulaire et invite à explorer plus avant les mécanismes en jeu dans l'inégal accès à la connaissance de la signification des mots.

\section{INDEX}

Mots-clés: Descripteurs TESE (2009) : classes sociales - compétences langagières, autres : activité définitionnelle - vocabulaire profond - sociolinguistique développementale

Keywords: TESE descriptors (2009) : social classes - language skills, others: definitional activity deep vocabulary - developmental sociolinguistics

\section{AUTHOR}

\section{THIERRY CHANSELME}

Université Clermont Auvergne - Ecole supérieure du Professorat de l'Education - Laboratoire ACTé < thierry.chanselme@orange.fr> 